\title{
Improvement of Responsivity of Unified Power Flow Controller in Digital Control System
}

\author{
Shin-ichi Hamasaki ${ }^{1}$, Shinya Miyazaki ${ }^{1}$, Wataru Shirota ${ }^{1}$, Mineo Tsuji ${ }^{1}$ \\ ${ }^{1}$ Division of Electrical Engineering and Computer Science, Nagasaki University, Japan \\ E-mail: hama-s@nagasaki-u.ac.jp
}

\begin{abstract}
The Unified Power Flow Controller (UPFC) can flexibly manage power flow and maintain line voltage. The UPFC consists of two inverters in parallel side and series side. In parallel side, the reactive power can be compensated to improve the power factor. In series side, the voltage drop can be compensated to maintain proper line voltage. It is necessary for the operation in both sides to output the current and the voltage quickly and accurately. As the method for the UPFC control, the deadbeat control is applied. The deadbeat control is able to realize a quick response of the current and voltage control for only a sampling period compared with the general PI control. A principle and simulation results are presented in this paper.
\end{abstract}

\section{INTRODUCTION}

In recent years, as distributed generation systems increase in the power line, energy conservation and stable supply of power is getting to be required. In the power transmission, power factor drop caused by the reactive component of the load and the voltage drop due to unexpected accident occur. The reduction of power quality becomes the problem. FACTS components which can flexibly control the power flow and line voltage are investigated and developed. The Unified Power Flow Controller (UPFC)[1]-[7] is one of the FACTS components. The UPFC consists of two inverters with a common DC capacitor and is able to perform the reactive power compensation and the voltage drop compensation. It is possible to improve the power quality by using the UPFC. On the other hand, a digital control system by software is applied to control the UPFC flexibly. In order to obtain good compensation performance of the UPFC in the digital system, it is necessary to control output current and voltage quickly and accurately. In this study, the deadbeat (DB) control[8][9] is applied to improve the responsivity. The deadbeat control is able to realize a quick response of the current and voltage control for only a sampling period compared with the general PI control. Theory of the proposed control method and simulation results are presented in this paper.

\section{CONTROL Method}

\section{A. Configuration of UPFC}

A circuit configuration of the UPFC is shown in Fig.1. The UPFC consists of two inverters with a common capacitor on DC bus and output filters on each AC bus. A block diagram of Parallel side with the deadbeat control is shown in Fig.2. In

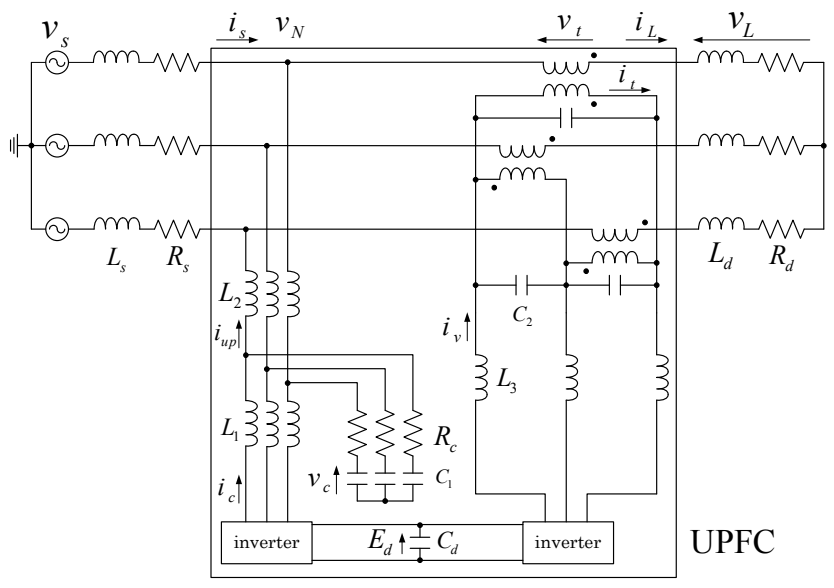

Fig. 1 Circuit configuration of UPFC

parallel side, the reactive power is compensated to improve the power factor. At the same time, in order to keep the voltage of the common DC capacitor, the output current for the active power is controlled by the parallel side inverter. The deadbeat control makes the current follow to the calculated current reference quickly and accurately.

A block diagram of series side with the deadbeat control is shown in Fig.3. In series side, the voltage drop is compensated to maintain proper line voltage. It is necessary to control with quick and accurate response because the control performance directly affects the compensation performance.

The deadbeat control is independently applied to regulate the output current in parallel side and output voltage in series side. Principle of the DB control is shown in Fig.4. In the proposed scheme, each output can be controlled by changing the output pulse width $\Delta T(k)$ of the inverter. It is determined from detected currents and voltages by a sampling period using the theoretical formula obtained from the state equation of the system.

\section{B. DB control of parallel side}

Fig.5 shows a single phase equivalent circuit of parallel side for analysis of the deadbeat control. Theory of single phase circuit is applied to three phase using $\alpha-\beta$ transform. The detail about it will be described in section $D$. 


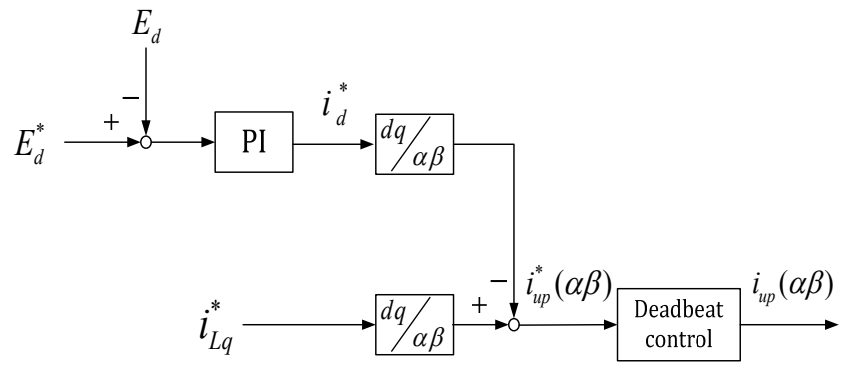

Fig.2 Block diagram of parallel side of the UPFC

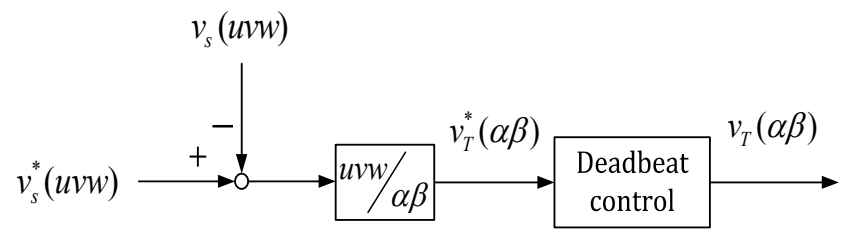

Fig.3 Block diagram of series side of the UPFC

The output pulse width can be obtained by calculating from the detected $i_{c}, i_{u p}, v_{c}$ and $v_{N} . v_{N}$, which is voltage of connected point, is regarded as a disturbance in the control of parallel side. When four parameters $i_{c}, v_{c}, i_{u p}$, and $v_{N}$ are selected as the states, the state equations are obtained as follows.

$$
\begin{aligned}
& \frac{d}{d t} \boldsymbol{x}_{\boldsymbol{1}}=\boldsymbol{A}_{\mathbf{1}} \boldsymbol{x}_{\mathbf{1}}+\boldsymbol{b}_{\boldsymbol{1}} v_{i 1} \\
& i_{u p}=\boldsymbol{c}_{\boldsymbol{1}} \boldsymbol{x}_{\boldsymbol{1}} \\
& \boldsymbol{x}_{\boldsymbol{1}}=\left[\begin{array}{l}
i_{c} \\
v_{c} \\
i_{u p} \\
v_{N}
\end{array}\right] \quad \boldsymbol{A}_{\mathbf{1}}=\left[\begin{array}{cccc}
-\frac{R_{c}}{L_{1}} & -\frac{1}{L_{1}} & \frac{R_{c}}{L_{1}} & 0 \\
\frac{1}{C_{1}} & 0 & -\frac{1}{C_{1}} & 0 \\
\frac{R_{c}}{L_{2}} & \frac{1}{L_{2}} & -\frac{R_{c}}{L_{2}} & -\frac{1}{L_{2}} \\
0 & 0 & 0 & 0
\end{array}\right] \quad \boldsymbol{b}_{\boldsymbol{1}}=\left[\begin{array}{c}
\frac{1}{L_{1}} \\
0 \\
0 \\
0
\end{array}\right] \\
& \boldsymbol{c}_{\boldsymbol{1}}=\left[\begin{array}{llll}
0 & 0 & 1 & 0
\end{array}\right]
\end{aligned}
$$

(1) and (2) can be converted into (3) and (4), which are expressions of the discrete time system using the output pulse width $\Delta T$ in Fig.4.

$$
\begin{aligned}
\boldsymbol{x}_{1}(k+1) & =\boldsymbol{F} \boldsymbol{x}_{1}(k)+\boldsymbol{g} \Delta T_{1}(k) \\
i_{u p}(k) & =\boldsymbol{c}_{1} \boldsymbol{x}_{1}(k)
\end{aligned}
$$

The matrixes $\boldsymbol{F}$ and $\boldsymbol{g}$ are calculated by (5), and become constant.

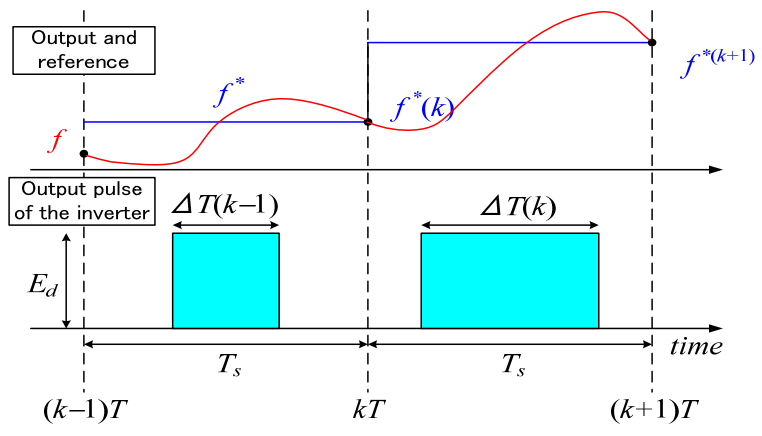

Fig.4. Principle of the DB control

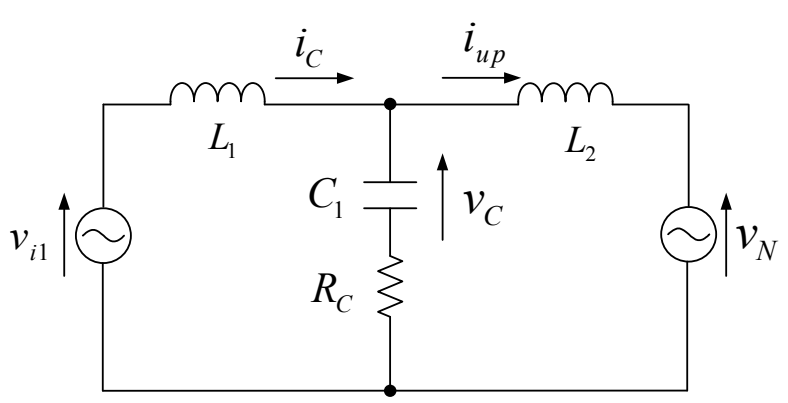

Fig.5 Analysis model of parallel side (single phase)

$$
\begin{gathered}
\boldsymbol{F}=e^{A_{1} \boldsymbol{T}_{s}}, \boldsymbol{g}=e^{\frac{A_{1} \boldsymbol{T}_{s}}{2}} \boldsymbol{b}_{1} E_{d} \\
\boldsymbol{F}=\left[\begin{array}{cccc}
F_{11} & F_{12} & F_{13} & F_{14} \\
F_{21} & F_{22} & F_{23} & F_{24} \\
F_{31} & F_{32} & F_{33} & F_{34} \\
0 & 0 & 0 & 1
\end{array}\right] \quad \boldsymbol{g}=\left[\begin{array}{l}
g_{1} \\
g_{2} \\
g_{3} \\
g_{4}
\end{array}\right]
\end{gathered}
$$

(6) is obtained from (3)-(5).

$$
i_{u p}(k+1)=F_{31} i_{c}(k)+F_{32} v_{c}(k)+F_{33} i_{u p}(k)+F_{34} v_{N}+g_{3} \Delta T_{1}(k)
$$

Here, the pulse width $\Delta T_{1}(k)$ can be calculated as (7) when $i_{u p}(k+1)$ is replaced to $i_{u p}{ }^{*}(k+1)$.

$$
\Delta T_{1}(k)=\frac{i_{u p}{ }^{*}(k+1)-F_{31} i_{c}(k)-F_{32} v_{c}(k)-F_{33} i_{u p}(k)-F_{34} v_{N}(k)}{g_{3}}
$$

When $\Delta T_{1}(k)$ is negative, the inverter DC voltage is given as $-\mathrm{E}_{\mathrm{d}}$ of width $\left|\Delta T_{1}\right|$. By outputting the calculated $\Delta T_{1}(k), i_{u p}$ $(k+1)=i_{u p}{ }^{*}(k+1)$ can be realized after a sample period. 


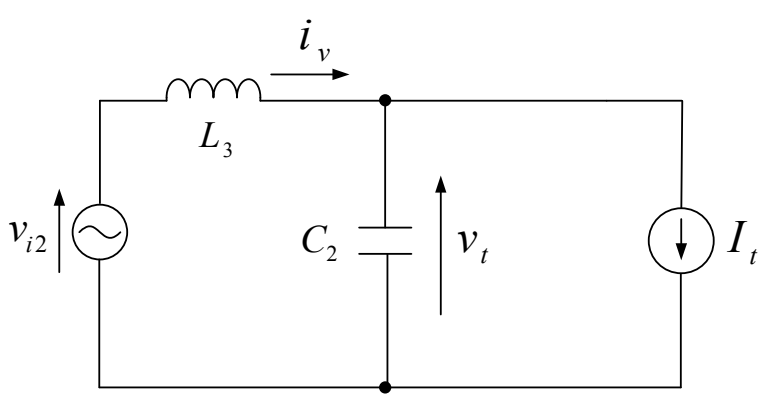

Fig.6 Analysis model of series side (single phase)

\section{DB control of series side}

Fig. 6 shows a single phase equivalent circuit of series side for analysis of the deadbeat control. The output pulse width is obtained by calculating from the detected $i_{v}, v_{t}$ and $I_{t} . I_{t}$, which is current of transformer depends on load current, is regarded as a disturbance in the control of series side. $v_{t}$ is primary voltage of transformer.

When three parameters $i_{v}, v_{t}$, and $I_{t}$ are selected as the states, the state equations are obtained as follows.

$$
\begin{aligned}
\frac{d}{d t} \boldsymbol{x}_{2} & =\boldsymbol{A}_{2} \boldsymbol{x}_{2}+\boldsymbol{b}_{2} v_{i 2} \\
v_{t} & =\boldsymbol{c}_{2} \boldsymbol{x}_{2}
\end{aligned}
$$

$$
\begin{aligned}
& \boldsymbol{x}_{2}=\left[\begin{array}{c}
v_{t} \\
i_{v} \\
I_{t}
\end{array}\right] \quad A_{2}=\left[\begin{array}{ccc}
0 & \frac{1}{3 C_{2}} & -\frac{1}{C_{2}} \\
-\frac{1}{L_{3}} & 0 & \frac{1}{L_{3}} \\
0 & 0 & 0
\end{array}\right] \quad \boldsymbol{b}_{2}=\left[\begin{array}{c}
0 \\
\frac{1}{L_{3}} \\
0
\end{array}\right] \\
& \boldsymbol{c}_{2}=\left[\begin{array}{lll}
1 & 0 & 0
\end{array}\right]
\end{aligned}
$$

(8) and (9) can be converted into (10) and (11) of the discrete time system by giving the output pulse width $\Delta T_{2}$ as show in Fig.4.

$$
\begin{gathered}
\boldsymbol{x}_{2}(k+1)=\boldsymbol{M} \boldsymbol{x}_{2}(k)+\boldsymbol{n} \Delta T_{2}(k) \\
v_{t}(k)=\boldsymbol{c}_{2} \boldsymbol{x}_{2}(k) \\
\boldsymbol{M}=e^{\boldsymbol{A}_{2} \boldsymbol{T}_{s}}, \boldsymbol{n}=e^{\frac{\boldsymbol{A}_{2} \boldsymbol{T}_{s}}{2}} \boldsymbol{b}_{2} E_{d}
\end{gathered}
$$

$$
\boldsymbol{M}=\left[\begin{array}{ccc}
M_{11} & M_{12} & M_{13} \\
M_{21} & M_{22} & M_{23} \\
0 & 0 & 1
\end{array}\right] \quad \boldsymbol{n}=\left[\begin{array}{l}
n_{1} \\
n_{2} \\
n_{3}
\end{array}\right]
$$

The matrixes $\boldsymbol{M}$ and $\boldsymbol{n}$ are calculated by (12) and become constant.

(13) is obtained from (10)-(12).

$v_{t}(k+1)=M_{11} v_{t}(k)+M_{12} i_{v}(k)+M_{13} I_{t}(k)+n_{1} \Delta T_{2}(k)$

Here, the pulse width $\Delta T(k)$ can be calculated in (14) when $v_{t}(k+1)$ equals to $v_{t}^{*}(k+1)$.

$$
\Delta T_{2}(k)=\frac{v_{t}^{*}(k+1)-M_{11} v_{t}(k)-M_{12} i_{v}(k)-M_{13} I_{t}(k)}{n_{1}}
$$

By outputting the calculated $\Delta T_{2}(k), v_{t}(k+1)=v_{t}{ }^{*}(k+1)$ can be realized after a sampling period.

\section{Output of three phase pulse}

(7) and (14) derived from Figs.5 and 6 are expressions in a single phase. Those can be extended to three phase. Three phase currents are transformed into $\alpha$ - $\beta$ components by (15).

$$
\left[\begin{array}{l}
i_{\alpha} \\
i_{\beta}
\end{array}\right]=\frac{2}{3}\left[\begin{array}{rrr}
1 & -\frac{1}{2} & -\frac{1}{2} \\
0 & \frac{\sqrt{3}}{2} & -\frac{\sqrt{3}}{2}
\end{array}\right]\left[\begin{array}{l}
i_{u} \\
i_{v} \\
i_{w}
\end{array}\right]
$$

Three phase voltages are also calculated by the same matrix of (15). And line-to-line voltages are transformed into $\alpha$ - $\beta$ components by (16).

$$
\left[\begin{array}{l}
v_{\alpha} \\
v_{\beta}
\end{array}\right]=\frac{2}{3}\left[\begin{array}{ccc}
\frac{1}{2} & 0 & -\frac{1}{2} \\
0 & \frac{\sqrt{3}}{2} & 0
\end{array}\right]\left[\begin{array}{l}
v_{u v} \\
v_{v w} \\
v_{w u}
\end{array}\right]
$$

All the detected currents and voltages for the control are transformed by (1) and (2) respectively. The $\alpha$ and $\beta$ components are independent without interference. Thus, each component can be treated as a single phase component and applied to the proposed DB control in (7) or (14). The pulse widths $\Delta T_{\alpha}$ and $\Delta T_{\beta}$ are determined from (7) and (14) respectively. The pulse width $\Delta T_{\alpha}$ and $\Delta T_{\beta}$ are converted into three phase pulse. (17) shows the transformation to the three 
phase pulse.
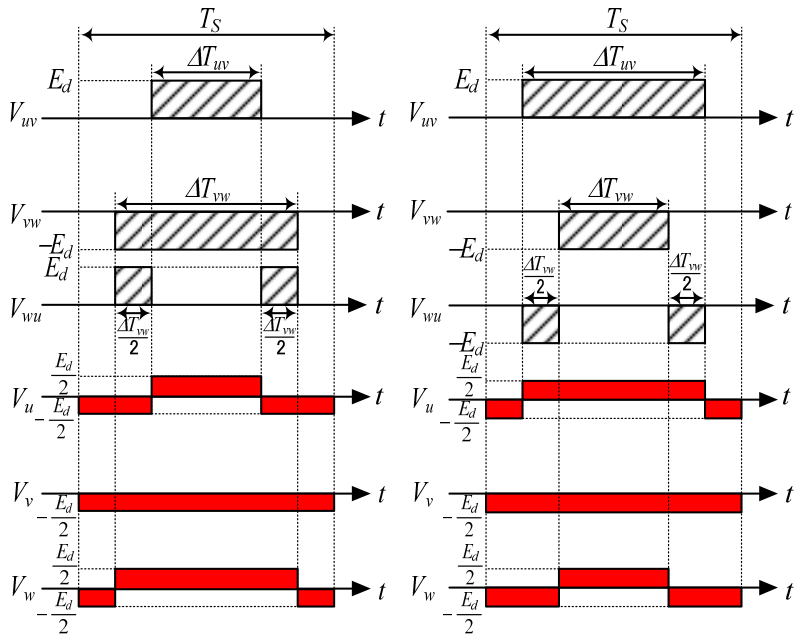

Fig.7 Principle of pulse conversion ( $\Delta T_{u v}>0$ and $\Delta T_{v w}<0$ )
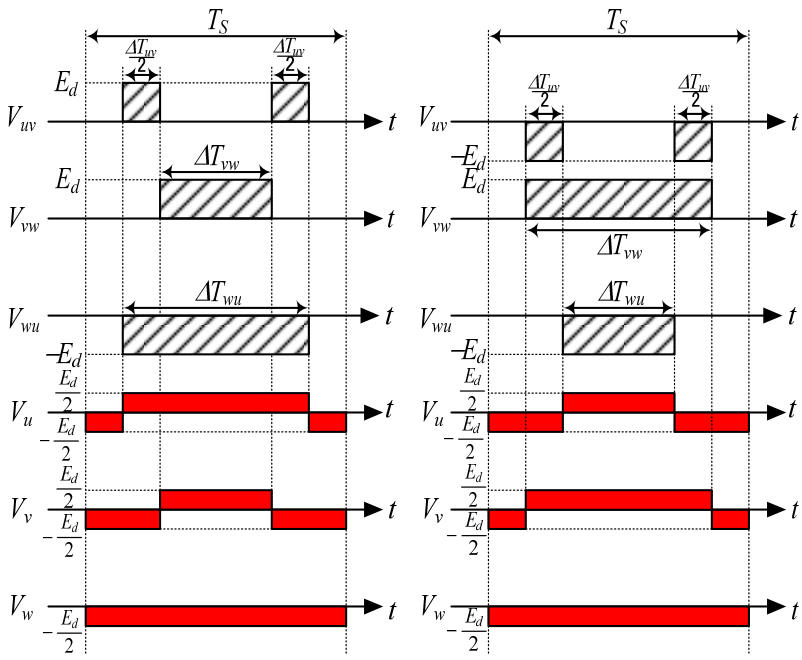

Fig.8 Principle of pulse conversion ( $\Delta T_{v w}>0$ and $\Delta T_{w u}<0$ )
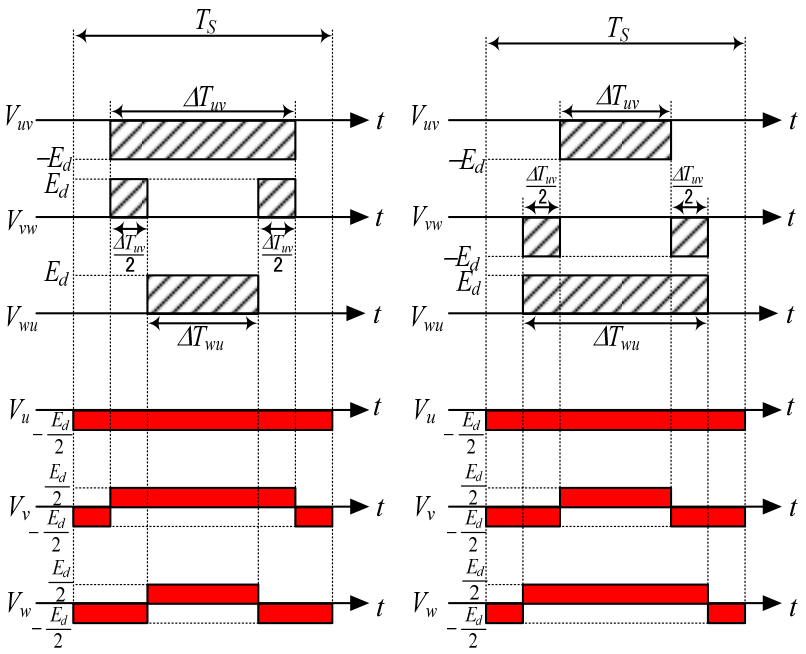

Fig.9 Principle of pulse conversion $\left(\Delta T_{w u}>0\right.$ and $\left.\Delta T_{u v}<0\right)$

$$
\left[\begin{array}{l}
\Delta T_{u v} \\
\Delta T_{v w} \\
\Delta T_{w u}
\end{array}\right]=\frac{3}{2}\left[\begin{array}{cc}
1 & -\frac{1}{\sqrt{3}} \\
0 & \frac{2}{\sqrt{3}} \\
-1 & -\frac{1}{\sqrt{3}}
\end{array}\right]\left[\begin{array}{l}
\Delta T_{\alpha} \\
\Delta T_{\beta}
\end{array}\right]
$$

Figs.7-9 show patterns of pulse output method. Each line voltage pulse is classified by combination of positive and negative pulses. There are three patterns, which are $\Delta T_{u v}>0$ and $\Delta T_{v w}<0, \Delta T_{v w}>0$ and $\Delta T_{w u}<0, \Delta T_{w u}>0$ and $\Delta T_{u v}<0$. Output of phase voltage is realized by the triangular PWM.

\section{SimUlation RESUlts}

Simulation is performed in Fig.1. Circuit parameters of the simulation are shown in Table.1. Simulation results in steady state are shown in Figs.10-17.

Figs.10-13 show the results of the general PI control. Figs.14-17 show the results of the proposed method. The proposed control compared with the general PI control, improvement of power factor and compensation of voltage drop can be performed properly by the UPFC operation. Result of the comparison, the DB control has a superior compensation performance than the PI control. In particular, the voltage control of the series side is much improved by the proposed control in Fig.17. In steady state, the DB control is able to follow to the current and the voltage reference quickly and accurately, and it has less time delay than the PI control.

Figs.18-21 show the transient response of the output current in the parallel side and the output voltage in the series side. In the transient response, the load increases to double at $0.3 \mathrm{~s}$. In the transient response, the PI control takes around 3 cycles to converge. However, the DB control is able to follow quickly for variations in load. In comparison of the transient response, the performance of the proposed control has conspicuous advantage.

\begin{tabular}{|lll|lll|}
\hline$L_{S}:$ & 0.4 & $(\mathrm{mH})$ & $R_{S}:$ & $0.1 \quad(\Omega)$ \\
\hline$L_{d}:$ & 8.0 & $(\mathrm{mH})$ & $R_{d}:$ & 4.0 & $(\Omega)$ \\
\hline$L_{1}:$ & 1.5 & $(\mathrm{mH})$ & $L_{2}:$ & 2.0 & $(\mathrm{mH})$ \\
\hline$C_{1}:$ & 5.0 & $(\mu \mathrm{F})$ & $R_{c}:$ & 2.0 & $(\Omega)$ \\
\hline$L_{3}:$ & 4.0 & $(\mathrm{mH})$ & $C_{2}:$ & 50.0 & $(\mu \mathrm{F})$ \\
\hline$E_{d}^{*}:$ & 200 & $(\mathrm{~V})$ & $V_{S}:$ & 100 & $(\mathrm{~V})$ \\
\hline
\end{tabular}




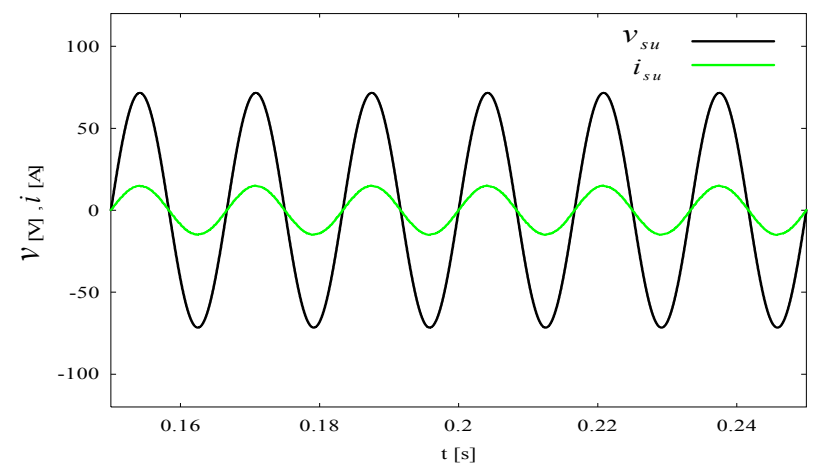

Fig.10 Line voltage $v_{s u}$ and line current $i_{s u}$ (by PI control)

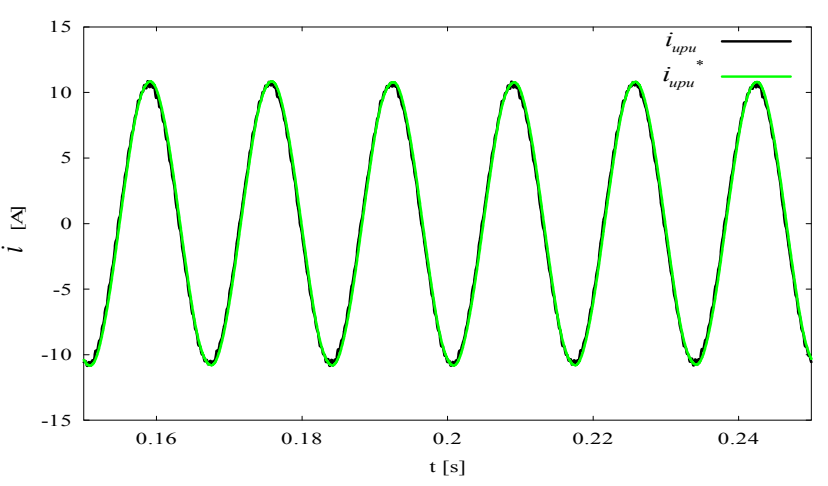

Fig.11 The output current $i_{u p}$ and reference $i_{u p}{ }^{*}$ in the parallel side (by PI control)

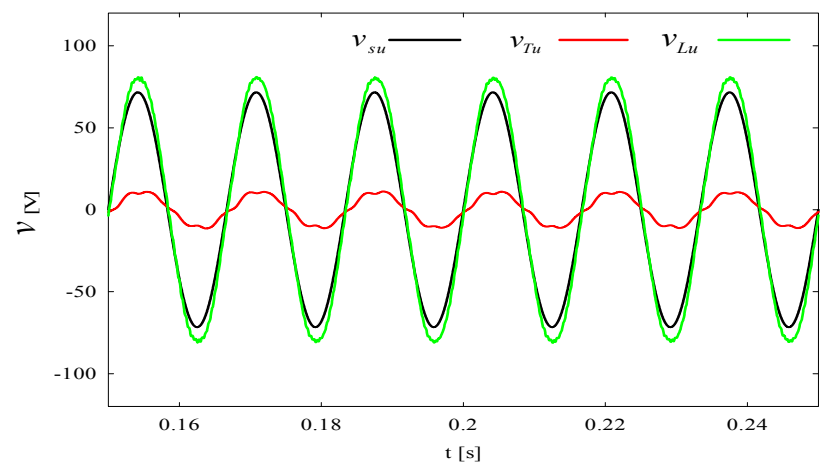

Fig.12 The voltage drop compensation (by PI control)

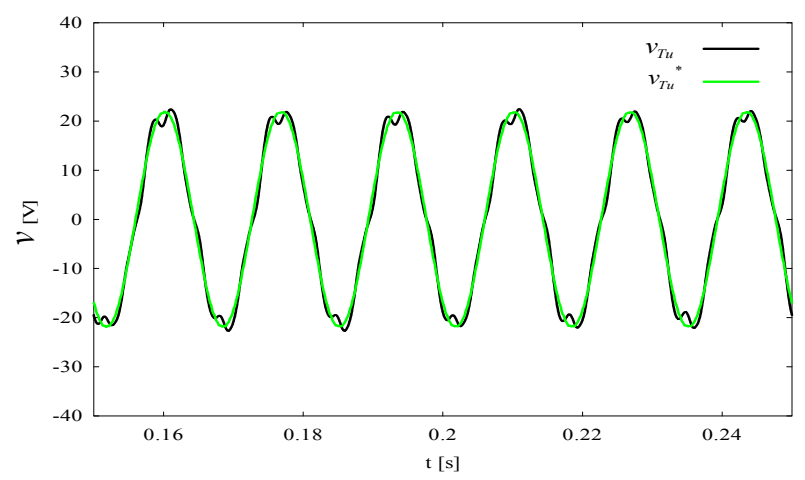

Fig. 13 The primary output voltage $v_{t}$ and reference $v_{t}{ }^{*}$ in the series side (by PI control)

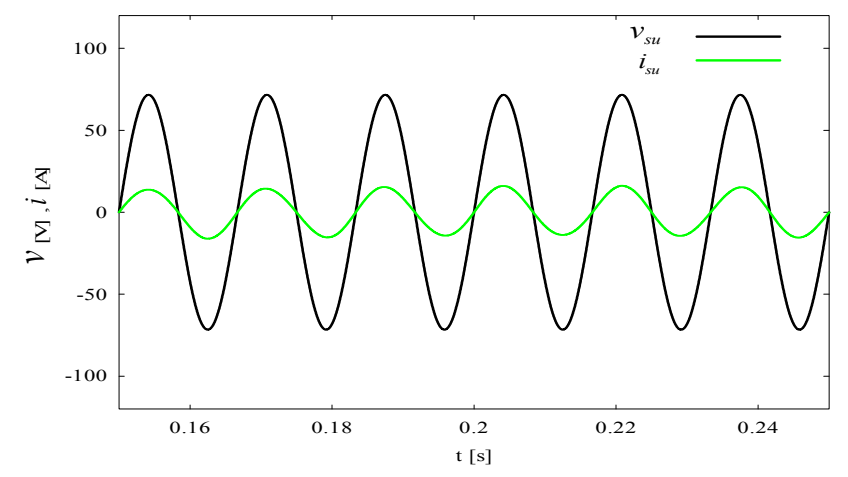

Fig. 14 Line voltage $v_{s u}$ and line current $i_{s u}$ (by DB control)

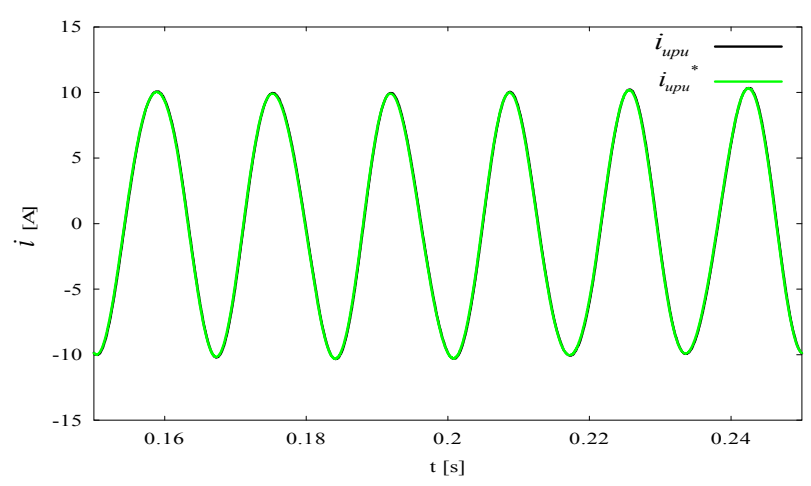

Fig. 15 The output current $i_{u p}$ and reference $i_{u p}{ }^{*}$ in the parallel side (by DB control)

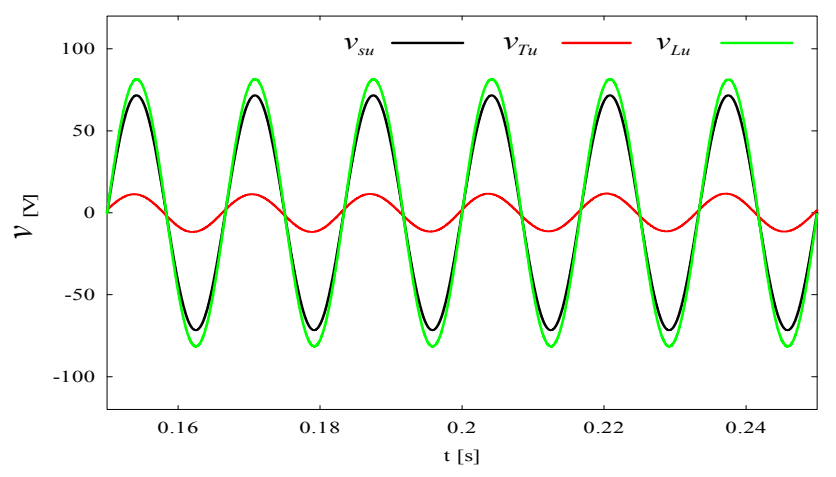

Fig.16 The voltage drop compensation (by DB control)

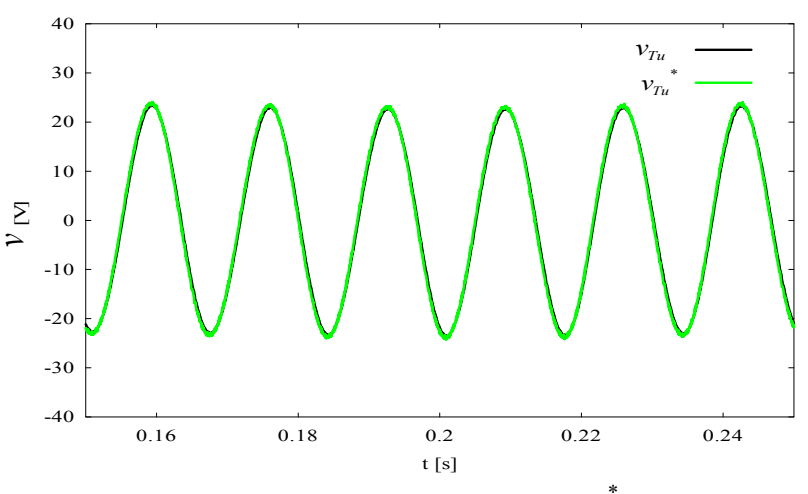

Fig. 17 The primary output voltage $v_{t}$ and reference $v_{t}{ }^{*}$ in the series side (by DB control) 


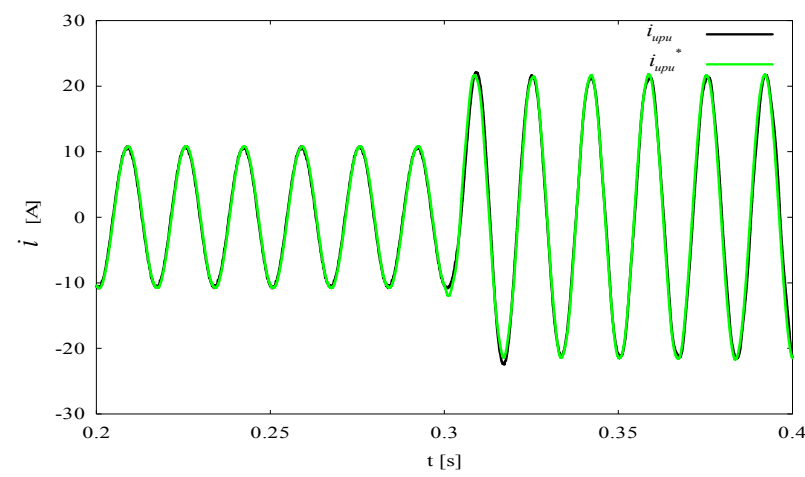

Fig.18 The transient response of the output current in the parallel side (by PI control)

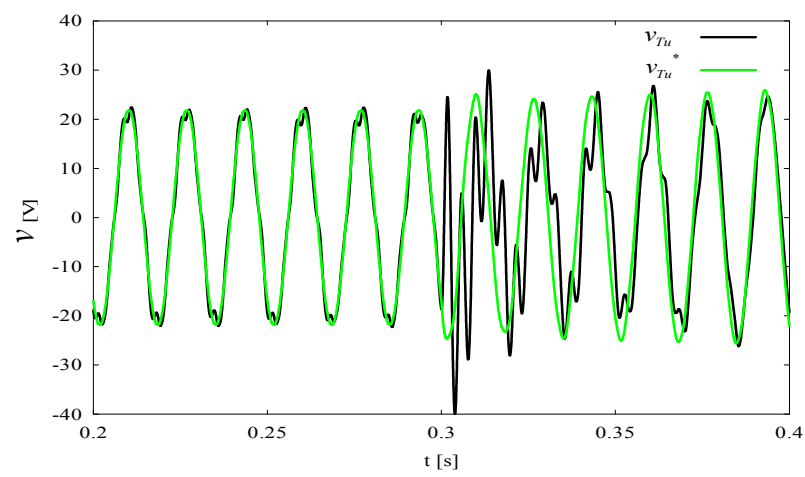

Fig.19 The transient response of the output voltage in the series side (by PI control)

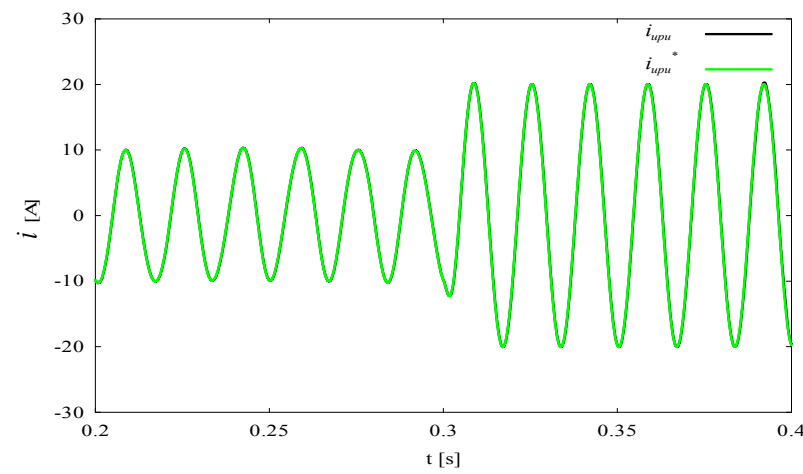

Fig.20 The transient response of the output current in the parallel side (by DB control)

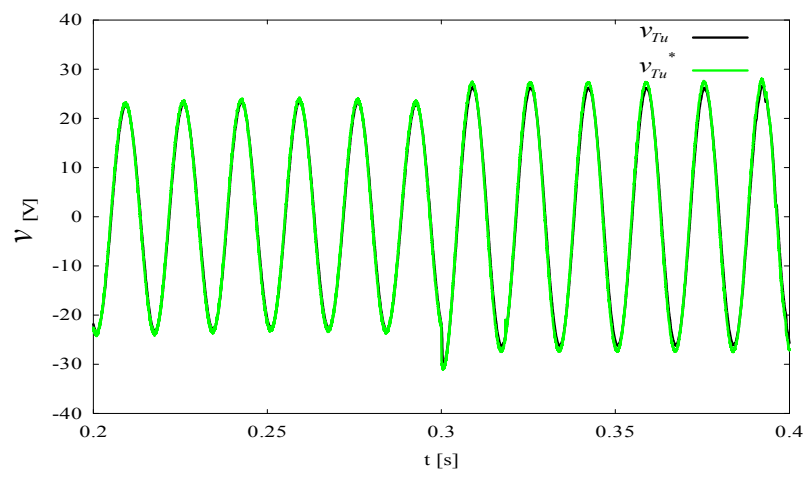

Fig. 21 The transient response of the output voltage in the series side (by DB control)

\section{CONCLUSION}

In this study, the DB control is proposed in order to improve performance of the UPFC and the effectiveness of the DB control is verified by comparison with the PI control. The DB control is able to realize a quick and accurate response of the current and voltage control for only a sampling period compared with the general PI control.

In simulation, the power quality is improved by performing the reactive power compensation and the voltage drop compensation in the UPFC. It is clarified that excellent results are obtained by the proposed DB control, which is able to operate quickly and accurately in steady state and transient response.

\section{REFERENCES}

[1] H. Fujita, Y. Watanabe and H. Akagi, "Control and Analysis of a Unified Power Flow Controller", IEEE Trans. on Power Electronics, Vol.14, No.6, pp.1021-1027 (1999)

[2] H. Fujita, H. Akagi and Y. Watanabe, "Dynamic Control and Performance of a Unified Power Flow Controller for Stabilizing an AC Transmission System", IEEE Trans. on Power Electronics, Vol.21, No.4, pp.1013-1020 (2006)

[3] Y. Taguchi, H. Akagi, "A Proposal of a Load/Source Simulatior Based on the UPFC and Operating Performance of a 200-V, 10-kW Laboratory System (In Japanese)", IEE of Japan Trans. on Industrial Application, Vol.123, No.11, pp.1295-1303 (2003)

[4] M.A. Sayed, N. Inayoshi, T. Takeshita, and F. Ueda, "Line Loss Minimization of Loop Distribution Systems Using UPFC", IEE of Japan Trans. on Industrial Application, Vol.128, No.4, pp-508-515 (2008)

[5] M.A. Sayed and T. Takeshita, "All Nodes Voltage Regulation and Line Loss Minimization in Loop Distribution Systems Using UPFC", IEEE Trans. on Power Electronics, Vol.26, No.6, pp.1694-1703 (2011)

[6] J. Wang and F.Z. Peng : "Unified Power Flow Controller Using the Cascade Multilevel Inverter", IEEE Trans. on Power Electronics, Vol.19, No.4, pp.1077-1084 (2004)

[7] B. Mwinyiwiwa, B. Liu and B-T. Ooi, "Multiterminal Unified Power Flow Controller", IEEE Trans. on Power Electronics, Vol.15, No.6, pp.1088-1093 (2000)

[8] S. Hamasaki, T. Kusaba, Y. Mazaki, M. Tsuji, "A Novel Method for Active Filter Applying The Deadbeat Control and The Repetitive Control", ICEMS2009, No. LS5B-2 (2009)

[9] S. Hamasaki and A. Kawamura, "Improvement of Current Regulation of Line-Current-Detection-type Active Filter based on Deadbeat Control", IEEE Trans. on Industrial Application, Vol.39, No.2, pp.536-541 (2003) 